\title{
Efficacy and Safety of Valsartan/Amlodipine Single-Pill Combination in 11,422 Chinese Patients with Hypertension: an Observational Study
}

Dayi $\mathrm{Hu} \cdot$ Lisheng Liu $\cdot$ Weimin Li

To view enhanced content go to www.advancesintherapy.com

Received: February 25, 2014 / Published online: July 2, 2014

(c) The Author(s) 2014. This article is published with open access at Springerlink.com

\section{ABSTRACT}

Introduction: Single-pill combination (SPC) therapy of two drugs is recommended by international guidelines, including the Chinese guidelines (2010), for the treatment of hypertension in high-risk patients who require marked blood pressure (BP) reductions. Realworld data on the efficacy and safety of valsartan/amlodipine (Val/Aml) SPC are scarce.

On behalf of the CHINA STATUS II study group.

The authors of the CHINA STATUS II study group are presented in the "Appendix".

Electronic supplementary material The online version of this article (doi:10.1007/s12325-014-0132-x) contains supplementary material, which is available to authorized users.

D. $\mathrm{Hu}(\bowtie)$

Department of Cardiology, Peking University

People's Hospital, Beijing 100044, China

e-mail: hudayi1@163.com;

dayi.hu@medmail.com.cn

L. Liu

Fuwai Hospital and Cardiovascular Institute, Chinese Academy of Medical Sciences, Beijing 100037, China

W. Li

Department of Cardiology, The First Affiliated Hospital of Harbin Medical University, Harbin 150001, China
The present study is the first observational study in China to evaluate the efficacy (primary endpoint) and safety of $\mathrm{Val} / \mathrm{Aml}(80 / 5 \mathrm{mg}) \mathrm{SPC}$ in Chinese patients with hypertension whose BP was not adequately controlled by monotherapy in a real-world setting.

Methods: This prospective, multicenter, openlabel, post-marketing observational study included 11,422 Chinese adults ( $\geq 18$ years) with essential hypertension from 238 sites of 29 provinces who were prescribed once-daily Val/Aml (80/5 mg) SPC. Patients were treated for 8 weeks. The primary efficacy variable of the study included changes in mean sitting systolic BP (MSSBP) and mean diastolic BP (MSDBP) from baseline to week 8 (end point). The secondary efficacy variable of the study included BP control rate and response rate at week 4 and 8 . Safety assessments included recording and measurement of all adverse events (AEs) and vital signs in the safety population.

Results: A significant reduction of $27.1 \mathrm{mmHg}$ in MSSBP (159.6 vs. $132.5 \mathrm{mmHg} ; P<0.0001$ ) and $15.2 \mathrm{mmHg}$ in MSDBP (95.6 vs. $80.4 \mathrm{mmHg} ; \quad P<0.0001)$ from baseline was observed at week 8 . The BP-lowering efficacy 
of Val/Aml SPC was independent of age and comorbidities. BP control of $<140 / 90 \mathrm{mmHg}$ was achieved in $76.8 \% \quad(n=8,692)$ of the patients. The most frequently reported AEs were dizziness $(0.2 \%)$, headache $(0.2 \%)$, upper respiratory tract infection $(0.2 \%)$, and edema $(0.2 \%)$. Only three serious AEs were reported and they were not drug-related.

Conclusion: This is the first evidence-based real-world data in Chinese hypertensive patients which demonstrate the efficacy and safety of $\mathrm{Val} / \mathrm{Aml}(80 / 5 \mathrm{mg})$ SPC.

Keywords: Amlodipine; Blood pressure; Cardiology; Hypertension; Single-pill combination; Valsartan

\section{INTRODUCTION}

Hypertension, an important risk factor for cardiovascular diseases (CVDs), affected an estimated one billion people worldwide in $2000[1,2]$. This number is expected to rise to 1.56 billion by 2025 [1]. In China, hypertension is the leading preventable risk factor for premature mortality [3]. The majority of cardiovascular (CV) deaths in China were attributed to hypertension (2.11 million) in 2005 [3]. The China National Nutrition and Health Survey findings indicated that nearly 0.2 billion (18\%) adults in 2002 had hypertension and only $6.1 \%$ of them achieved blood pressure (BP) control [4]. In 2010, a survey conducted among Chinese adults aged $\geq 18$ years revealed that the prevalence of hypertension was $33.5 \%$ [5].

According to the Chinese guidelines (2010) for the management of hypertension, a reduction in systolic BP (SBP) and diastolic BP (DBP) less than $140 / 90 \mathrm{mmHg}$ in all hypertensive patients, and less than
$130 / 80 \mathrm{mmHg}$ in patients with concomitant diabetes or chronic kidney disease (CKD) is essential to reduce the risk of CV mortality [6]. However, evidence from a previous study suggests that only a small proportion of the hypertensive population in China receives adequate treatment or achieves effective BP control [7].

Single-drug therapy or monotherapy is the preferred way to initiate hypertension treatment, but BP goals $(<140 / 90 \mathrm{mmHg})$ cannot always be achieved with single-drug therapy in patients who require strong BP reductions $[8,9]$. It has been proposed that in high-risk hypertensive patients who require a rapid and pronounced BP control, single-pill combination (SPC) or fixed-dose combination can be beneficial as a first-line therapy [10]. SPCs offer several advantages such as improved efficacy, reduced adverse events (AEs), and improved patient compliance compared with monotherapy [8]. Safe and effective combination of two or more drug classes is recommended for the majority of patients with hypertension to achieve their BP goal, which may translate into reduced risk of $\mathrm{CV}$ outcomes [8-14]. The 2013 European Society of Hypertension/European Society of Cardiology Guidelines for the management of arterial hypertension recommend the initiation of hypertension treatment with a combination of two drugs from different classes in patients with stage 2 hypertension (SBP $\geq 160 \mathrm{mmHg}$ or DBP $\geq 100 \mathrm{mmHg}$ ) [15]. Moreover, use of an antihypertensive drug combination in a single pill may further enhance BP control by reducing pill burden and enhancing compliance $[8,16]$.

Amlodipine (Aml) and valsartan (Val) are widely used antihypertensive drugs that have also been established to improve $\mathrm{CV} /$ renal outcomes [17, 18]. The combination of Val and Aml has shown an additive BP-lowering 
effect and minimal AEs in adult patients with mild-to-moderate hypertension compared to individual monotherapies [13, 14, 19]. The efficacy and safety of this combination have also been demonstrated in the Chinese population through randomized controlled clinical trials [20-22]. Two of these studies evaluated SPC therapy and showed that it provided better outcomes in terms of efficacy, safety, and tolerability in Chinese patients compared with monotherapy [20, 21]. However, the effectiveness of Val/Aml SPC therapy has not been evaluated in real-world conditions in China. The present observational study was conducted to evaluate the effectiveness and safety of the Val $(80 \mathrm{mg})$ and Aml (5 mg) SPC therapy on BP control at week 8 in a population of Chinese patients with uncontrolled BP on antihypertensive monotherapy in a real-life practice setting.

\section{METHODS}

\section{Study Design}

This was a multicenter, post-marketing, prospective observational study enrolled adult patients with essential hypertension, whose BP was not adequately controlled by monotherapy. Patients from 238 regional centers across 29 provinces of China were enrolled from October 12, 2010 to February 20, 2012. The Val/Aml (80/ $5 \mathrm{mg}$ ) SPC once-daily dosing was prescribed to the patients instead of the previous antihypertensive drugs. The treatment was in compliance with the routine clinical outpatient practice in China. The duration of the study was 8 weeks with a follow-up at every 4 weeks. If a patient did not achieve BP control at the end of 4 weeks, an additional antihypertensive agent could be added.

\section{Study Population}

The study population included adult Chinese patients with essential hypertension [(mean sitting SBP (MSSBP) $\geq 140 \mathrm{mmHg}(\geq 130 \mathrm{mmHg}$ for diabetes or CKD) and/or mean sitting DBP (MSDBP) $\geq 90 \mathrm{mmHg}$ ) ( $\geq 80 \mathrm{mmHg}$ for diabetes or CKD)], whose BP was not adequately controlled by monotherapy as mentioned in the Val/Aml package insert approved by the State Food and Drug Administration.

Patients were selected based on the following inclusion criteria: male or female aged $\geq 18$ years with essential hypertension; on oral antihypertensive monotherapy (angiotensinconverting enzyme inhibitors (ACEIs), angiotensin-II receptor blockers (ARBs), calcium channel blockers (CCBs), diuretics, or $\beta$-blockers); MSSBP $\geq 140 \mathrm{mmHg}$ and/or MSDBP $\geq 90 \mathrm{mmHg}$; with diabetes or CKD, MSSBP $\geq 130 \mathrm{mmHg}$ and/or MSDBP $\geq 80 \mathrm{mmHg}$. Patients were prescribed the Val/Aml SPC based on the clinical judgment of the investigators according to the patient's condition and taking into consideration the package insert; signed informed consent was obtained for all patients. The exclusion criteria included women who were pregnant or lactating and or of child-bearing potential without adequate contraception measures, and or any other conditions which precluded administration of the drug based on the investigator's discretion.

Patient demographics, history of hypertension, past medical history and present complications, BP measure, present monotherapy, and the reason for switching medication were recorded for all the participants during the initial visit.

The study was conducted in accordance with the International Conference on Harmonization-Good Clinical Practice 
(ICH-GCP) and applicable local regulations in China. The study received approval from the Ethical Review Committee of The First Hospital of Harbin Medical University. All procedures followed were in accordance with the ethical standards of the responsible committee on human experimentation (institutional and national) and with the Helsinki Declaration of 1975, as revised in 2000 and 2008. Informed consent was obtained from all patients for being included in the study.

\section{Efficacy Assessments}

The primary efficacy variable of the study included changes in MSSBP and MSDBP from baseline to week 8 (end point). The secondary efficacy variable of the study included BP control rate and response rate (defined as the percentage of patients achieving MSSBP $<140 \mathrm{mmHg}$ or MSSBP reduction $\geq 20 \mathrm{mmHg}$ from baseline; MSDBP $<90 \mathrm{mmHg}$ or MSDBP reduction $\geq 10 \mathrm{mmHg}$ from baseline) at week 4 and 8. A subgroup analysis of the reduction in MSSBP and MSDBP values from baseline was performed at week 8 based on age $(<65,65-<80$, $\geq 80)$, baseline SBP $(<140,140-159,160-179$, $\geq 180$ ), different CV risk factors (dyslipidemia, coronary heart disease, diabetes, heart failure (HF), stroke, and kidney disease), and antihypertensive treatments (ACEIs, ARBs, $\beta$ blockers, CCBs, and diuretics).

\section{Safety Assessments}

Safety assessments included recording and measurement of all AEs and vital signs in the safety population. The incidence of AEs was recorded at week 4 and 8 of the study period. Each AE was defined by its duration, severity, and relationship to the study drug.

\section{Statistical Analysis}

Full analysis set (FAS) included patients with at least one post-baseline efficacy evaluation, and safety set (SS) included patients with at least one post-baseline safety evaluation. The FAS was used for all efficacy analyses. All statistical analyses were performed using SAS ${ }^{\circledR}$ Software version 9.2 (SAS Institute Inc., Cary, NC, USA) at two-sided significance level $(P)$ of $<0.05$. Demographic and baseline variables were summarized using descriptive statistics, including the mean, standard deviation (SD), median, minimum and maximum values for numeric variables, and the count number and percentage for categorical variables. Paired $t$ test, two-way analysis of variance, the Chi square test, and logistic regression were used to analyze efficacy end points. The BP control rates and medication compliance among subgroups was analyzed using Chi square test and Wilcoxon rank-sum test, respectively. AEs were summarized by incidence rates (frequencies and percentages). The efficacy, tolerability, and medication compliance of the SPC evaluated by the investigator and the patient were classified as 'very good', 'good', 'general', and 'not good'.

\section{RESULTS}

\section{Demographic and Baseline Characteristics}

A total of 11,422 patients were enrolled for the study, of which 27 patients were excluded for violating GCP, 71 patients did not meet the inclusion criteria, and 63 patients withdrew. Finally, 11,312 patients were included in the FAS, and 11,321 patients in the SS (Fig. 1). The detailed demographic and baseline characteristics of the patients are presented in Table 1 . The mean age of the patients included 
in the FAS was 58.4 years. Approximately $57 \%$ of the patients were male; and $97.1 \%$ of the patients belonged to the Han ethnic group. The mean duration of hypertension was $8.3 \pm 7.3$ years with a mean baseline SBP/DBP value of $159.6 / 95.6 \mathrm{mmHg}$. A large proportion of the patients in this study had hypertension without any other CV risk factors (36.9\%; $n=4,174)$.

CCB $(47.9 \% ; n=5,413)$ was the most commonly used monotherapy prior to enrollment, followed by ARB (25.2\%; $n=2,853)$ and ACEI (15.3\%; $n=1,728)$. The most frequent reasons for switching to SPC included patients unable to achieve BP goals on either the initial or titrated dose of monotherapy (82.0\% and $15.0 \%$, respectively), or could not tolerate the original therapy (3.2\%).

\section{Efficacy Results}

\section{Primary Efficacy}

$\mathrm{Val} / \mathrm{Aml}$ SPC resulted in a significant reduction in MSSBP by $20.1 \mathrm{mmHg}$ from baseline after 4 weeks (159.6 vs. $139.5 \mathrm{mmHg} ; \quad P<0.0001)$ and $27.1 \mathrm{mmHg}$ after 8 weeks (159.6 vs. $132.5 \mathrm{mmHg} ; \quad P<0.0001)$ of treatment. In addition, a significant reduction in MSDBP by $10.6 \mathrm{mmHg}$ from baseline after 4 weeks (95.6 vs.

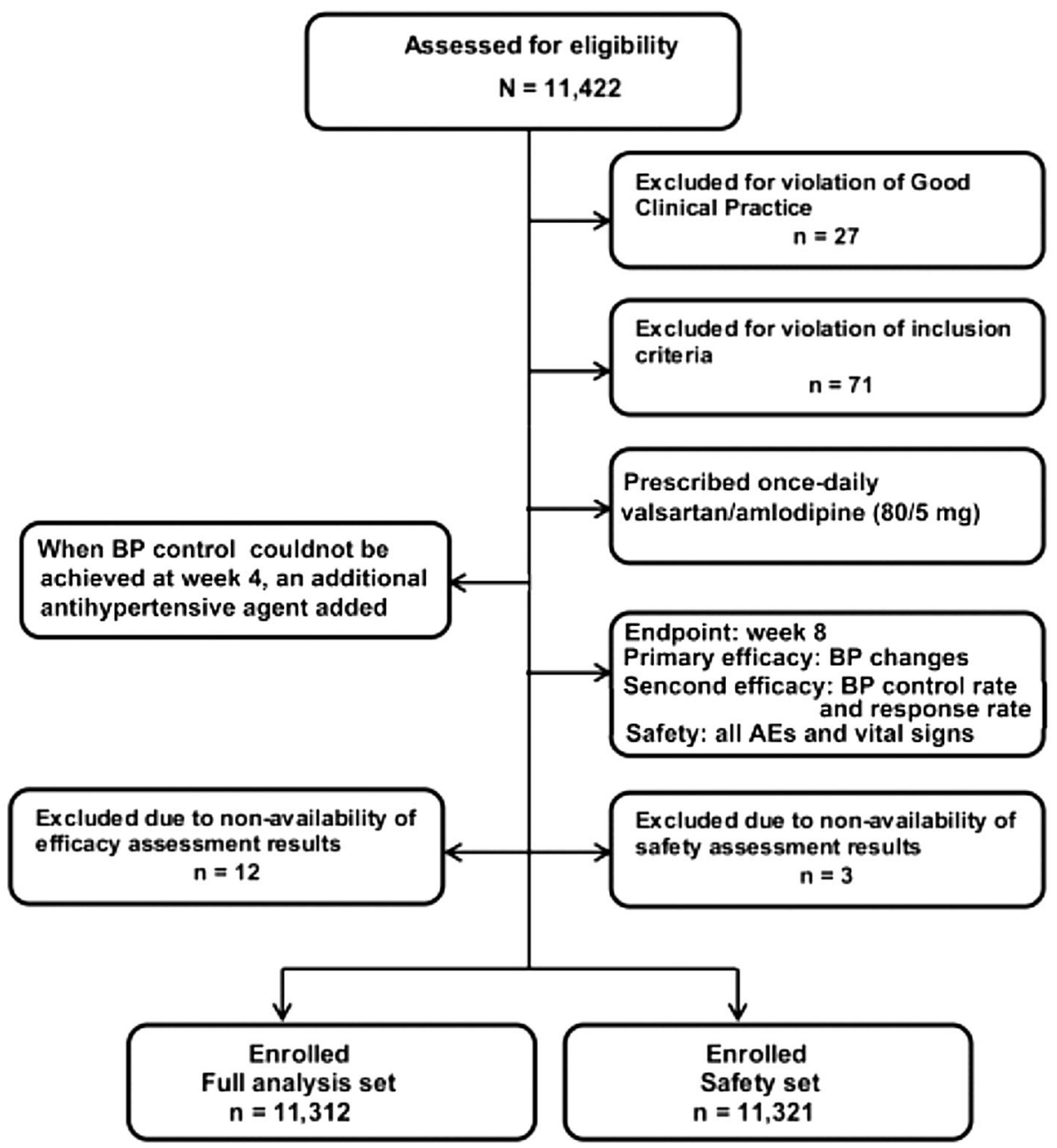

Fig. 1 Patient flow chart. $B P$ blood pressure 
Table 1 Demographic and baseline characteristics of the patients $(n=11,312)$

\begin{tabular}{|c|c|}
\hline Variable & Baseline values \\
\hline \multicolumn{2}{|l|}{ Age (years) } \\
\hline Mean age (SD) & $58.4(13.8)$ \\
\hline \multicolumn{2}{|l|}{ Patients in each group, $n(\%)$} \\
\hline$<65$ & $7,677(67.9)$ \\
\hline $65-<80$ & $2,863(25.3)$ \\
\hline$\geq 80$ & $746(6.6)$ \\
\hline \multicolumn{2}{|l|}{ Gender, $n(\%)$} \\
\hline Male & $6,456(57.1)$ \\
\hline Mean height (SD), $\mathrm{cm}$ & $167.15(7.8)$ \\
\hline Mean weight (SD), kg & $69.18(11.4)$ \\
\hline \multicolumn{2}{|l|}{ Mean waistline (SD), cm } \\
\hline Female & $82.1(11.0)$ \\
\hline Male & $89.3(10.4)$ \\
\hline \multicolumn{2}{|l|}{ Ethnicity, $n(\%)$} \\
\hline Han & $10,989(97.1)$ \\
\hline \multicolumn{2}{|l|}{ BMI $\left(\mathrm{kg} / \mathrm{m}^{2}\right)$} \\
\hline Mean BMI (SD) & $24.7(3.2)$ \\
\hline \multicolumn{2}{|l|}{ Patients in each group, $n(\%)$} \\
\hline Underweight (BMI <19) & $266(2.4)$ \\
\hline Normal $(19 \leq \mathrm{BMI}<24)$ & $4,449(39.3)$ \\
\hline Overweight $(24 \leq \mathrm{BMI}<28)$ & $5,127(45.3)$ \\
\hline Obese $(\mathrm{BMI}>28)$ & $1,447(12.8)$ \\
\hline \multicolumn{2}{|c|}{ Present cardiovascular risk factors/medical history, $n(\%)$} \\
\hline Dyslipidemia & $2,702(23.9)$ \\
\hline Diabetes & $1,865(16.5)$ \\
\hline $\mathrm{CHD}$ & $1,753(15.5)$ \\
\hline HF & $189(1.7)$ \\
\hline Stroke & $591(5.2)$ \\
\hline Kidney disease & $355(3.1)$ \\
\hline \multicolumn{2}{|l|}{ BP data $(\mathrm{mmHg})$} \\
\hline Mean baseline SBP & 159.6 \\
\hline Mean baseline DBP & 95.6 \\
\hline
\end{tabular}

Table 1 continued

\begin{tabular}{ll}
\hline Variable & Baseline values \\
\hline $\begin{array}{l}\text { Patients in each SBP group }(n, \%) \\
<140\end{array}$ & $184(1.6)$ \\
$140-159$ & $5,398(47.7)$ \\
$159-180$ & $4,816(42.6)$ \\
$\geq 180$ & $914(8.1)$ \\
Previous antihypertensive drug classes, $n(\%)$ \\
$\beta$-Blockers & $903(7.99)$ \\
CCBs & $5,413(47.90)$ \\
ACEIs & $1,728(15.29)$ \\
Diuretics & $321(2.84)$ \\
ARB & $2,853(25.2)$ \\
Others & $82(0.73)$ \\
Unknown & $12(0.11)$ \\
\hline
\end{tabular}

$A C E I s$ angiotensin-converting enzyme inhibitors, $A R B s$ angiotensin-II receptor blockers, $B M I$ body mass index, $B P$ blood pressure, $C C B s$ calcium channel blockers, $C H D$ chronic heart disease, $D B P$ diastolic blood pressure, $H F$ heart failure, $S B P$ systolic blood pressure

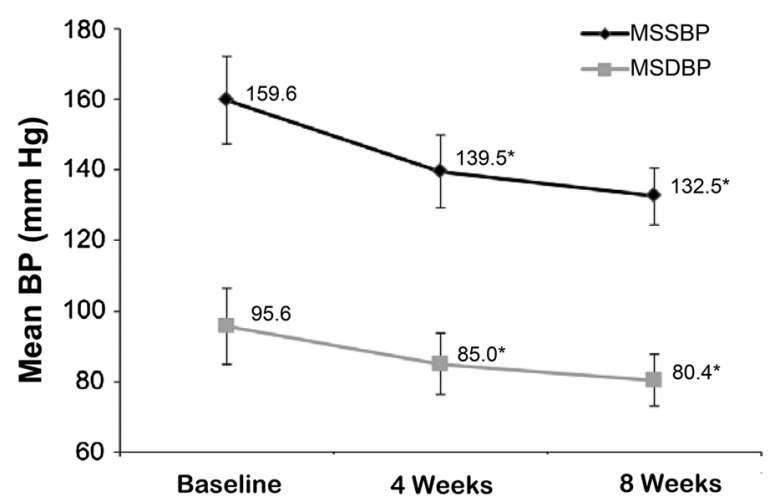

Fig. 2 Reduction in mean systolic and diastolic BP after 4 and 8 weeks of valsartan/amlodipine single-pill combination treatment. ${ }^{*} P<0.0001$ vs. baseline. Error bars represent standard deviation. $B P$ blood pressure, $M S D B P$ mean sitting diastolic blood pressure, MSSBP mean sitting systolic blood pressure

$85.0 \mathrm{mmHg} ; P<0.0001)$ and $15.2 \mathrm{mmHg}$ after 8 weeks (95.6 vs. $80.4 \mathrm{mmHg} ; P<0.0001$ ) of treatment was also noted (Fig. 2). 


\section{Efficacy in Different Subgroups}

The patients were grouped based on their age as $<65$ years, $65-<80$ years, and $\geq 80$ years. The mean BP-lowering efficacy of Val/Aml SPC was independent of age (Fig. 3). Another subgroup analysis based on baseline SBP levels (i.e., SBP $<140, \quad 140-159$, 160-179, and $\geq 180 \mathrm{mmHg}$ ) showed significant reductions in MSSBP and MSDBP $(P<0.0001)$ from baseline in all the baseline SBP groups after 8 weeks of treatment (Fig. 4). The mean BP reduction increased with an increase in baseline SBP levels.

The mean reduction in MSSBP from baseline at week 8 in patients treated with $\mathrm{Val} / \mathrm{Aml}$ from the different antihypertensive monotherapy groups, namely, $\beta$ blockers, CCBs, ACEIs, diuretics, ARBs, and others, was 28.3, 27.3, $28.1,27.4,25.7$, and $31.9 \mathrm{mmHg}$, respectively (Fig. 5). Reduction in MSDBP was also achieved in the different monotherapy groups after 8 weeks of treatment. As depicted in Fig. 6, after 8 weeks of SPC treatment, the mean reduction in MSSBP and MSDBP was also observed in the subgroups with different $\mathrm{CV}$ risk factors.

\section{Secondary Efficacy}

A BP control of $<140 / 90 \mathrm{mmHg}$ was achieved in $76.8 \%(n=8,692)$ of patients after 8 weeks of $\mathrm{Val} / \mathrm{Aml}$ SPC treatment. After 8 weeks of treatment, $98.0 \%$ of patients $(n=11,084)$ responded to treatment. Of the 11,312 patients in this study, only $686(6.1 \%)$ patients required add-on therapy at 4 weeks in addition to the Val/Aml SPC treatment. Diuretics were the most frequently used add-on therapy during the study, and 14 patients added 2 additional antihypertensive drugs.

\section{Safety}

The SS consisted of 11,321 patients. The most frequently reported AEs were dizziness (0.2\%), headache $(0.2 \%)$, upper respiratory tract infection $(0.2 \%)$, and edema (0.2\%; Table 2$)$. Only three serious AEs were reported during the study; none of them were suspected to be related to the treatment. The tolerability of Val/Aml SPC as evaluated by investigators and patients was quite similar; both evaluations were rated as 'very good' by $63.3 \%(n=7,170)$ and $61.3 \%$

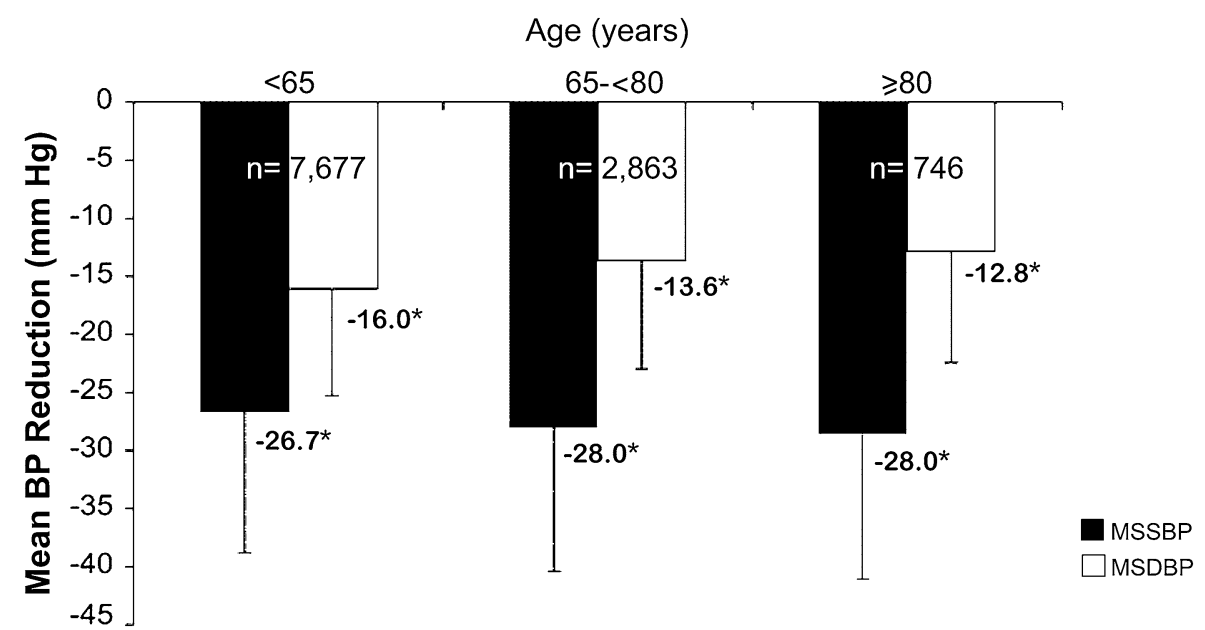

Fig. 3 MSSBP and MSDBP reduction in different age groups at week $8 .{ }^{*} P<0.0001$ vs. baseline. Error bars represent standard deviation. $B P$ blood pressure, $M S D B P$ mean sitting diastolic blood pressure, MSSBP mean sitting systolic blood pressure 


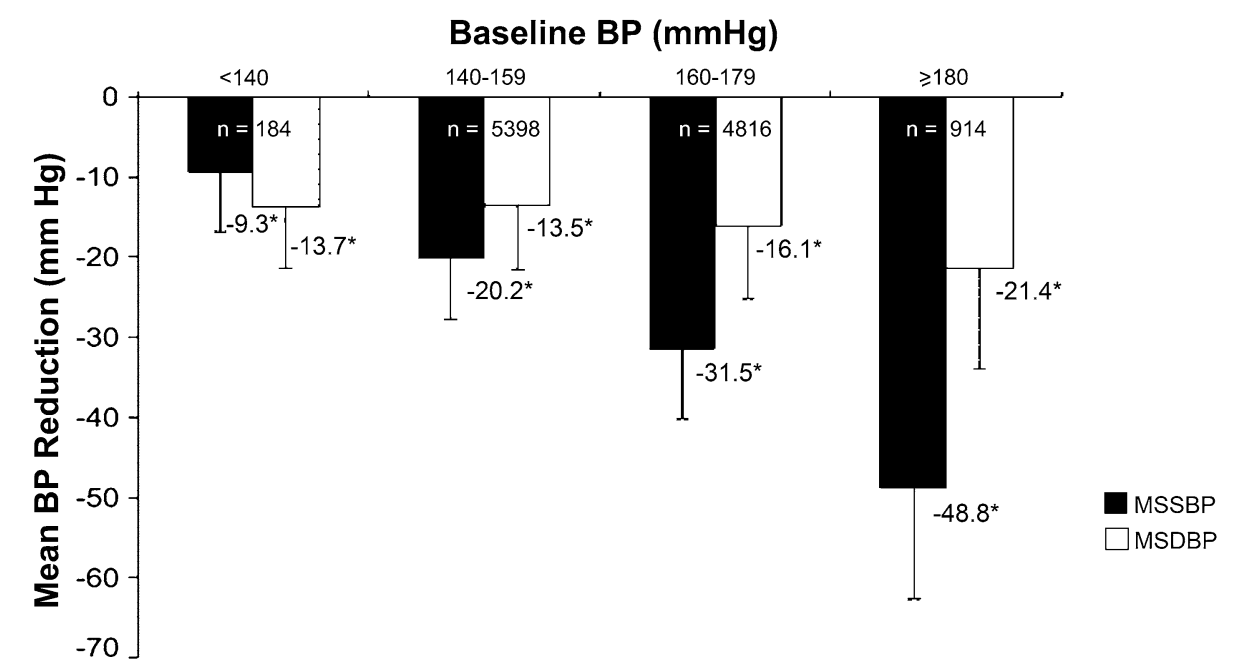

Fig. 4 MSSBP and MSDBP reduction in different baseline SBP subgroups at week $8 .{ }^{*} P<0.0001$ vs. baseline. Error bars represent standard deviation. $B P$ blood pressure,
MSDBP mean sitting diastolic blood pressure, MSSBP mean sitting systolic blood pressure, SBP systolic blood pressure

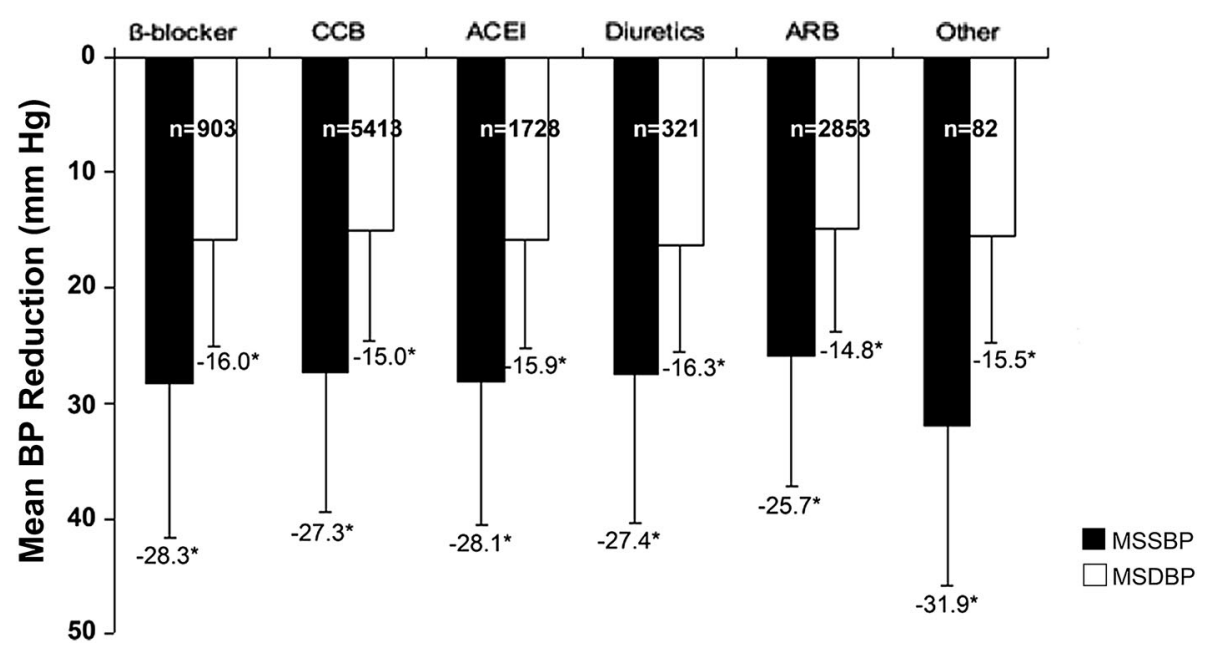

Fig. 5 Mean MSSBP and MSDBP reduction in different monotherapy subgroups at week $8 .{ }^{*} P<0.0001$ vs. baseline. $A C E I$ ace inhibitor, $A R B$ angiotensin receptor blocker, $B P$

$(n=6,942)$, respectively. According to the investigators' report, $86.9 \%(9,787$ of 11,266$)$ of the patients had a high adherence rate of $80 \%$ or greater while taking the Val/Aml SPC.

\section{DISCUSSION}

The present study is the first evidence-based, multicenter, observational real-world study that blood pressure, $C C B$ calcium channel blocker, $M S D B P$ mean sitting diastolic blood pressure, MSSBP mean sitting systolic blood pressure

demonstrates the effectiveness and safety of $\mathrm{Val} / \mathrm{Aml}$ SPC in reducing BP in a very large number of Chinese hypertensive patients. This study also showed that Val/Aml SPC was well tolerated with a good safety profile, even in patients aged 80 years and older (safety data not presented by age group). The prevalence rate of hypertension is on the rise in China, due to lack of awareness and treatment in addition to 


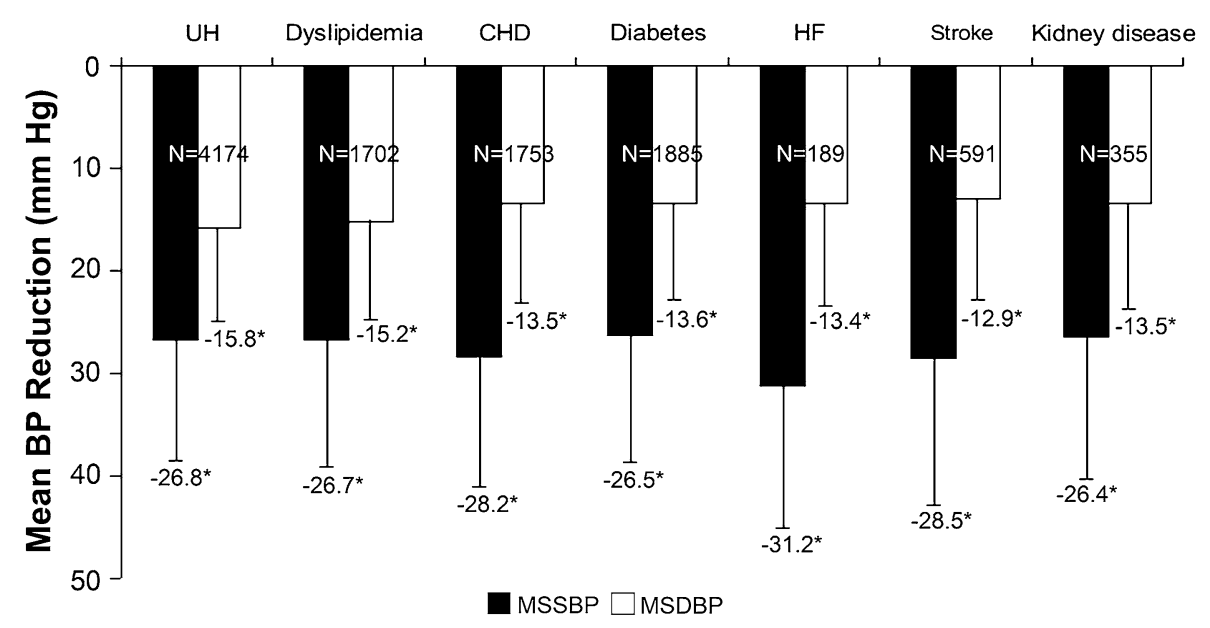

Fig. 6 MSSBP and MSDBP reduction in subgroups with different cardiovascular risk factors at week $8 .{ }^{*} P<0.0001$ vs. baseline. $B P$ blood pressure, $C H D$ coronary heart disease, $H F$ heart failure, $M S D B P$ mean sitting diastolic

Table 2 Incidence of adverse events

\begin{tabular}{lc}
\hline Variable & $\begin{array}{l}\text { Patients } \\
(\boldsymbol{n}=\mathbf{1 1 , 3 2 1})\end{array}$ \\
\hline Any AE, $n(\%)$ & $164(1.4)$ \\
Any SAE, $n(\%)$ & $3(<0.1)$ \\
AE related to treatment, $n(\%)$ & $64(0.6)$ \\
SAE related to treatment, $n(\%)$ & $0(0.0)$ \\
Discontinuations due to AEs, $n(\%)$ & $22(0.2)$ \\
Most frequent AEs, $n(\%)$ & \\
Dizziness & $27(0.2)$ \\
Headache & $25(0.2)$ \\
Edema & $19(0.2)$ \\
Upper respiratory tract infection & $18(0.2)$ \\
Cough & $9(0.1)$ \\
\hline
\end{tabular}

$A E$ adverse event, $S A E$ serious adverse event

inadequate control of the disease [7]. Various randomized clinical trials (RCTs) have studied the efficacy and safety of Val/Aml SPC in the Chinese population with essential hypertension $[20,22,23]$. However, most RCTs exclude patients with very high $\mathrm{BP}$ and those with a blood pressure, $M S S B P$ mean sitting systolic blood pressure, $S B P$ systolic blood pressure, $U H$ uncomplicated hypertension

risk of CVD and hence, do not represent findings from actual clinical practice [24]. In this observational study, patients with SBP $\geq 180 \mathrm{mmHg}$, and also those with different CV risk factors were included. Moreover, large observational studies allow the enrollment of an adequate number of patients for subgroup analyses.

Monotherapy is a rational therapeutic approach in patients with mild $\mathrm{CV}$ risk or mild BP elevation [25]. However, in about 70\% of patients, it is ineffective in achieving BP goals [25]. The use of combination therapy having complementary mechanisms of action is more effective than single agents in achieving optimal BP control $[8,26]$. A SPC of Val and $\mathrm{Aml}$ has been well tolerated regardless of age, sex, race, or ethnicity $[27,28]$. The safety and efficacy of this combination have also been demonstrated in RCTs of Chinese individuals $[20,21]$. In the present observational study, SPC therapy with $\mathrm{Val}$ and Aml resulted in significant reductions in MSSBP and MSDBP from baseline after 8 weeks, consistent with results of randomized controlled studies, including those 
conducted in Chinese patients [20-23, 29-33]. After 8 weeks of treatment, BP control $(<140 /$ $90 \mathrm{mmHg}$ ) with $\mathrm{Val} / \mathrm{Aml}$ combination was achieved in approximately $77 \%$ of the patients. This is similar to the significant BP control rate $(72.7 \%)$ observed in a broad spectrum of patients from eight countries (France, Spain, Belgium, Norway, Switzerland, Slovakia, Canada, and The United States) whose BP was initially uncontrolled by monotherapy and who were switched to Val/Aml 160/10 mg or 160/5 mg [31]. Likewise, another randomized controlled study in a general Asian population reported similar BP control rates $(69.2 \%)$ in patients treated with Val/Aml SPC (80/5 mg) [22]. Our findings support the efficacy of the $\mathrm{Val} / \mathrm{Aml}(80 / 5 \mathrm{mg}) \mathrm{SPC}$ in a real-world scenario as well.

SPC therapies improve medication adherence due to reduced pill burden $[8,34]$. In this study, as reported by the patients, more than $85 \%$ of patients took $80 \%$ or more of the $\mathrm{Val} / \mathrm{Aml}$ SPC, which could be one of the reasons for improved efficacy. This reinforces the fact that SPC therapy could improve adherence, which is an important factor that influences BP-lowering efficacy of the drug [35].

Combination therapy has the potential for attenuation of certain drug class-specific AEs [2]. Val/Aml SPC was well tolerated in this study. There was a low incidence of AEs reported in each treatment phase, with three serious AEs occurring; none of them was suspected to be related to study treatment. A common $\mathrm{AE}$ of CCBs is peripheral edema which occurs due to arteriolar dilatation, causing intracapillary hypertension and fluid extravasation [36]. Despite the use of a CCB (i.e., Aml) in the treatment phase, the incidence of peripheral edema was low with the SPC treatment. This may be attributed to a short observational period, a different dose, or minimization of CCB-induced edema by the ARB (i.e., Val) [2]. Likewise, a randomized controlled 8-week study of 349 Asian hypertensive patients (predominantly Chinese) treated with $\mathrm{Val} / \mathrm{Aml} 80 / 5$ reported no incidences of peripheral edema [22] and a similar study of 308 Asian patients reported a $1.3 \%$ incidence [23].

In this study, treatment was effective regardless of baseline $\mathrm{BP}$, comorbidities, monotherapy, or age. Elderly patients with hypertension are at an increased risk of adverse CVD outcomes such as HF and stroke [37]. Therefore, effective lowering of BP in the elderly is clinically very important. Clinical trials very rarely recruit from the elderly population, however, in the present observational study, a sufficient number of elderly patients over 80 years of age $(n=746)$ were included. In this study, after 8 weeks of Val/Aml SPC treatment reported significant reductions in BP in elderly patients. A recent RCT involving 61 Chinese elderly patients has also shown the efficacy of the $\mathrm{Val} / \mathrm{Aml}$ combination therapy in lowering $\mathrm{BP}$ in the elderly population [38].

A limitation of the study design was the addition of Val/Aml onto other antihypertensive monotherapies at week 0 or another antihypertensive drug added onto Val/ Aml at week 4 (when BP cannot be controlled), which might have contributed to increased efficacy of the treatment, although the impact of this has not been determined. This study observed a low incidence of AEs compared to several randomized trials with the same SPC, which may be attributed to the shorter treatment period of 8-weeks [13, 14]. Alternatively, it is possible that inadequate communication between the investigator and the patient could have resulted in non-recording of events and hence the low incidence of AEs. 


\section{CONCLUSION}

In conclusion, the findings from the first realworld observational study in Chinese hypertensive patients confirm the effectiveness and safety of using SPC Val and Aml in a large population. By initiating treatment with this combination, significant and early reductions in both MSSBP and MSDBP were achieved, which enabled a large proportion of the patients to reach a BP goal of $140 / 90 \mathrm{mmHg}$ in 8 weeks. The outcomes of this SPC were found to be safe and tolerable with low incidences of AEs in a wide range of Chinese patients with uncontrolled BP on monotherapy. Additional studies of longer durations are necessary to investigate the potential clinical benefits of this SPC on organ protection and CV outcome improvement.

\section{ACKNOWLEDGMENTS}

Sponsorship for this study and article processing charges was funded by Novartis Pharmaceuticals (China). The authors acknowledge Jack Zhang (MD), Xinhai Qiu (MD), Junsheng Ding and Yao Li of Novartis Pharma Co Ltd, Beijing, China, for their support in developing the protocol, Yusong $\mathrm{He}(\mathrm{MD})$ and Danni Shi (MD) of Novartis Pharma Co Ltd, Beijing, China, for their support in accomplishing the critical review, and ascertaining the scientific and factual accuracy of the manuscript. The authors also acknowledge Amit Bhat (PhD) and Salini Vasantha (PhD), Indegene Lifesystems Pvt. Ltd., for providing the necessary writing assistance and editorial support towards the development of the manuscript. This support was funded by Novartis.
Hu Dayi, Liu Lisheng and Li Weimin designed this clinical trial, analyzed the data and wrote the paper; Hu Dayi also contributed to data collection. All named authors meet the ICMJE criteria for authorship for this manuscript, take responsibility for the integrity of the work as a whole, and have given final approval to the version to be published. All authors had full access to all of the data in this study and take complete responsibility for the integrity of the data and accuracy of the data analysis.

Conflict of interest. Dayi $\mathrm{Hu}$, Lisheng Liu, and Weimin Li declare no conflict of interest.

Compliance with ethics guidelines. The study was conducted in accordance with the International Conference on HarmonizationGood Clinical Practice (ICH-GCP) and applicable local regulations in China. The study received approval from the Ethical Review Committee of The First hospital of Harbin Medical University. All procedures followed were in accordance with the ethical standards of the responsible committee on human experimentation (institutional and national) and with the Helsinki Declaration of 1975, as revised in 2000 and 2008. Informed consent was obtained from all patients for being included in the study.

Funding. Sponsorship for this study and article processing charges was funded by Novartis Pharmaceuticals (China).

Open Access. This article is distributed under the terms of the Creative Commons Attribution Noncommercial License which permits any noncommercial use, distribution, and reproduction in any medium, provided the original author(s) and the source are credited. 


\section{APPENDIX: CHINA STATUS II STUDY GROUP LIST}

Weiming Li, Ying Gao, Haitao Li, Yiqun Yao, Shenghua Ding, Lianyou Liu, Le Shi, Fengshi Tian, Hongtao Li, Zhiyuan Dong, Jian Wei, Xiaofei Wang, Ling Zhang, Lu Li, Ze Jin, Tiemin Jiang, Hua Ge, Qin Yu, Yili Ma, XianXi Gao, Xiaoyun Zhang, Xuebin Cao, Yanning Zhang, Lining Miao, Yixiu Liu, Haitao Li, Ping Yang, Yonghong Gao, Bin Wang, Xiaobo Zhou, Hongyun Zang, Xinping Du, Jianmin Li, Guodong Ma, Yuean Cao, Zheng Qu, Yu Wu, Hongmei Li, Yujie Zhou, Juhui An, Wei Wang, Chunfang Zhang, Hui Li, Chunling Dong, Yan Cui, Yang Wang, Shujuan Cheng, Wen Yu, Yuming Hao, Hongyu Wang, Lijun Wang, Zhicheng Song, Huimin Zhang, Xian Wang, Mingsheng Wang, Junli Gong, Lin Pi, Senbao Ma, Zhixing Wang, Lina Wang, Yang Zheng, Zhigang Li, Hongliang Cong, Xiaoyuan Lin, Ming Yang, Jian Kong, Guixia Wang, Hongbin Zou, Chengzhi Lu, Dawei Zang, Wenjie Yang, $\mathrm{Yu}$ Liu, Hongfang Liu, Yushen Xu, Guang Huang, Junduo Liu, Xiaoming Zhuang, Hongtao Yang, Xin Qi, Lin Wang, Aili Jiang, Mingzhi Long, Xiaoping Ji, Weidong Jin, Shuhong Ren, Shuzhen Han, Zuyou Shen, Yun Wu, Changwu Ruan, Jianrong Zhao, Meixiang Xiang, Jinyuan Zhang, Yufei $\mathrm{Wu}$, Xiaolan Li, Yong Zhou, Ping Li, Zhongchen Li, Xinbo Yang, Xiuming Zhang, Xianliang Zhang, Jiandong Ding, Hui Jin, Limin Sun, Zhijun Zhu, Jing Wang, Xingwei Zhang, Aizhen Li, Shengxiang Liu, Ying Liu, Guofang Cui, Feng Wang, Xiaochun $\mathrm{Wu}$, Wanhua $\mathrm{Hu}, \mathrm{Yu} \mathrm{Li}$, Guangzhi Cao, Xiangyang Liu, Wenjin Hua, Lingyun Yu, Lianhua Zhou, Shoulin Zhang, Ying Wang, Hongmei Guo, Ning Gu, Pingjin Gao, Chu Zhang, Ningfu Wang, Xiong Li, Yun Zhang, Shuwei Huang, Baorong Zhang, Guohai Su, Jian Zhou, Xiaohua Guo, Zhiyuan Song, Yongjian
Yang, Xiaohua Zhao, Mei Yu, Lixia Yang, Jianping Sun, Jianhong Tao, Yongbin Song, Liman Ai, Xianning Zhao, Huiqin Chen, Shenglin Zhang, Baili Zhou, Xiao Pang, Hua Zhang, Haicheng Zhang, Xuetao Cheng, Xingkai Zhang, Shaohua Li, Huading Zhou, Yun Lv, Zengying Wu, Bing Chen, Liqin Zhi, Guifang Liu, Xinling Wang, Yuanyuan Feng, Aidong Zhu, Jian Wang, Shouzhong Yang, Lu Wang, Chao Wang, Huaimin Guan, Guangshu Cheng, Zhishen Zhao, Fanliang Meng, Jian Wang, Guoli Wang, Hongmei Li, Ruiping Zhao, Junming Liu, Xianjun Xue, Hengliang Wang, Hao Wang, Zhigang Zhao, Chuanyu Gao, Chen Lei, Xinjuan Xu, Xiaohong Sang, Yulan Zhao, Ye Gu, Youzhi $\mathrm{Hu}$, Yulong Shi, Yongcheng Li, Fafei Zheng, Linqun $\mathrm{Hu}$, Xiaoming Lin, Yong Zhang, Bei Cheng, Bihui Zheng, Yumin Wang, Shaomin $\mathrm{Hu}$, Shangquan Xiong, Guoying Zhu, Yi Lin, Jinzhong Yang, Cuntai Zhang, Wuwang Fang, Ting Jiang, Bo Yang, Zhiliang He, Guoliang Jia, Yuerong Yang, Xiaomei Guo, Zicheng Li, Qiang Xie, Rong Lin, Xinqing Du, Yujun Ding, Shugen Xu, Boshang $\mathrm{Xu}$, Qiaoke Zheng, Yuhua Liao, Jianshe Liu, Yan Wang, Chun Xiao, Tiansong Wang, Guogang Zhang, Dong Wang, Yaling Huang, Yichang Guan, Xiaobo Gai, Jianqiu Liang, Xiaoling Peng, Yuanchun Zhang, Longgen Xiong, Li Yan, Yongxin Cai, Yong Yuan, Lurong Hong, Jun Lan, Shaohong Dong, Yugang Dong, Hui Chen, Tianlun Yang, Ying Ye.

\section{REFERENCES}

1. Kearney PM, Whelton M, Reynolds K, et al. Global burden of hypertension: analysis of worldwide data. Lancet. 2005;365:217-23.

2. Krzesinski JM, Cohen EP. Exforge (amlodipine/ valsartan combination) in hypertension: the evidence of its therapeutic impact. Core Evid. 2010;4:1-11. 
3. He J, Gu D, Chen J, et al. Premature deaths attributable to blood pressure in China: a prospective cohort study. Lancet. 2009;374: $1765-72$.

4. Wu Y, Huxley R, Li L, et al. Prevalence, awareness, treatment, and control of hypertension in China: data from the China National Nutrition and Health Survey 2002. Circulation. 2008;118:2679-86.

5. Li YC, Wang LM, Jiang Y, et al. Prevalence of hypertension among Chinese adults in 2010. Zhonghua Yu Fang Yi Xue Za Zhi. 2012;46:409-13.

6. Liu LS. Writing group of Chinese guidelines for the management of $\mathrm{H}$ (2010 Chinese guidelines for the management of hypertension). Zhonghua Xin Xue Guan Bing Za Zhi. 2011;39:579-615.

7. Gu D, Reynolds K, Wu X, et al. Prevalence, awareness, treatment, and control of hypertension in china. Hypertension. 2002;40:920-7.

8. Bangalore S, Shahane A, Parkar S, et al. Compliance and fixed-dose combination therapy. Curr Hypertens Rep. 2007;9:184-9.

9. Sica DA. Rationale for fixed-dose combinations in the treatment of hypertension: the cycle repeats. Drugs. 2002;62:443-62.

10. Karpov Y, Dongre N, Vigdorchik A, et al. Amlodipine/valsartan single-pill combination: a prospective, observational evaluation of the reallife safety and effectiveness in the routine treatment of hypertension. Adv Ther. 2012; 29:134-47.

11. Brachmann J, Ansari A, Mahla G, et al. Effective and safe reduction of blood pressure with the combination of amlodipine $5 \mathrm{mg}$ and valsartan $160 \mathrm{mg}$ in hypertensive patients not controlled by calcium channel blocker monotherapy. Adv Ther. 2008;25:399-411.

12. Destro M, Luckow A, Samson M, et al. Efficacy and safety of amlodipine/valsartan compared with amlodipine monotherapy in patients with stage 2 hypertension: a randomized, double-blind, multicenter study: the EX-EFFeCTS study. J Am Soc Hypertens. 2008;2:294-302.

13. Flack JM, Calhoun DA, Satlin L, et al. Efficacy and safety of initial combination therapy with amlodipine/valsartan compared with amlodipine monotherapy in black patients with stage 2 hypertension: the EX-STAND study. J Hum Hypertens. 2009;23:479-89.

14. Philipp T, Smith TR, Glazer R, et al. Two multicenter, 8-week, randomized, double-blind, placebo-controlled, parallel-group studies evaluating the efficacy and tolerability of amlodipine and valsartan in combination and as monotherapy in adult patients with mild to moderate essential hypertension. Clin Ther. 2007;29:563-80.

15. Hypertension EETFftMoA. 2013 Practice guidelines for the management of arterial hypertension of the European Society of Hypertension (ESH) and the European Society of Cardiology (ESC): ESH/ESC task force for the management of arterial hypertension. J Hypertens. 2013;31:1925-38.

16. Bramley TJ, Gerbino PP, Nightengale BS, et al. Relationship of blood pressure control to adherence with antihypertensive monotherapy in 13 managed care organizations. J Manag Care Pharm. 2006;12:239-45.

17. Julius S, Kjeldsen SE, Weber M, et al. Outcomes in hypertensive patients at high cardiovascular risk treated with regimens based on valsartan or amlodipine: the VALUE randomised trial. Lancet. 2004;363:2022-31.

18. Dahlof B, Sever PS, Poulter NR, et al. Prevention of cardiovascular events with an antihypertensive regimen of amlodipine adding perindopril as required versus atenolol adding bendroflumethiazide as required, in the angloscandinavian cardiac outcomes trial-blood pressure lowering arm (ASCOT-BPLA): a multicentre randomised controlled trial. Lancet. 2005;366:895-906.

19. Schunkert H, Glazer RD, Wernsing M, et al. Efficacy and tolerability of amlodipine/valsartan combination therapy in hypertensive patients not adequately controlled on amlodipine monotherapy. Curr Med Res Opin. 2009;25: 2655-62.

20. Ke YN, Huang J, Zhu JR, et al. Efficacy and safety of the single pill combination of valsartan $80 \mathrm{mg}$ plus amlodipine $5 \mathrm{mg}$ in mild to moderate essential hypertensive patients without adequate blood pressure control by monotherapy. Zhonghua Xin Xue Guan Bing Za Zhi. 2009;37:794-9.

21. Zhu D, Yang K, Sun N, et al. Amlodipine/valsartan $5 / 160 \mathrm{mg}$ versus valsartan $160 \mathrm{mg}$ in Chinese hypertensives. Int J Cardiol. 2013;167:2024-30.

22. Ke $\mathrm{Y}$, Zhu D, Hong $\mathrm{H}$, et al. Efficacy and safety of a single-pill combination of amlodipine/valsartan in Asian hypertensive patients inadequately controlled with amlodipine monotherapy. Curr Med Res Opin. 2010;26:1705-13.

23. Huang J, Sun NL, Hao YM, et al. Efficacy and tolerability of a single-pill combination of amlodipine/valsartan in Asian hypertensive 
patients not adequately controlled with valsartan monotherapy. Clin Exp Hypertens. 2011;33: 179-86.

24. Smith TR, Philipp T, Vaisse B, et al. Amlodipine and valsartan combined and as monotherapy in stage 2 , elderly, and black hypertensive patients: subgroup analyses of 2 randomized, placebo-controlled studies. J Clin Hypertens (Greenwich). 2007;9: 355-64.

25. Mancia G, De Backer G, Dominiczak A, et al. 2007 Guidelines for the management of arterial hypertension: the task force for the management of arterial hypertension of the European society of hypertension (ESH) and of the European society of cardiology (ESC). J Hypertens. 2007;25:1105-87.

26. Ferri C, Croce G, Desideri G. Role of combination therapy in the treatment of hypertension: focus on valsartan plus amlodipine. Adv Ther. 2008;25:300-20.

27. Calhoun DA, Lacourciere Y, Chiang YT, et al. Triple antihypertensive therapy with amlodipine, valsartan, and hydrochlorothiazide: a randomized clinical trial. Hypertension. 2009;54:32-9.

28. Fogari R, Zoppi A, Mugellini A, et al. Efficacy and safety of two treatment combinations of hypertension in very elderly patients. Arch Gerontol Geriatr. 2009;48:401-5.

29. Poldermans D, Glazes R, Kargiannis S, et al. Tolerability and blood pressure-lowering efficacy of the combination of amlodipine plus valsartan compared with lisinopril plus hydrochlorothiazide in adult patients with stage 2 hypertension. Clin Ther. 2007;29:279-89.

30. Hu DY, Liu LS, Yu JM, et al. National survey of blood pressure control rate in Chinese hypertensive outpatients-China STATUS. Zhonghua Xin Xue Guan Bing Za Zhi. 2010;38:230-8.
31. Allemann Y, Fraile B, Lambert M, et al. Efficacy of the combination of amlodipine and valsartan in patients with hypertension uncontrolled with previous monotherapy: the exforge in failure after single therapy (EX-FAST) study. J Clin Hypertens (Greenwich). 2008;10:185-94.

32. Trenkwalder $\mathrm{P}$, Schaetzl $\mathrm{R}$, Borbas $\mathrm{E}$, et al. Combination of amlodipine $10 \mathrm{mg}$ and valsartan $160 \mathrm{mg}$ lowers blood pressure in patients with hypertension not controlled by an ACE inhibitor/ CCB combination. Blood Press Suppl. 2008;2: 13-21.

33. RL. Exforge: a new combination to treat hypertension. Pharma Note. 2008; 23:1-11.

34. Bangalore S, Kamalakkannan G, Parkar S, et al. Fixed-dose combinations improve medication compliance: a meta-analysis. Am J Med. 2007;120: 713-9.

35. Gupta AK, Arshad S, Poulter NR. Compliance, safety, and effectiveness of fixed-dose combinations of antihypertensive agents: a metaanalysis. Hypertension. 2010;55:399-407.

36. Makani H, Bangalore S, Romero J, et al. Peripheral edema associated with calcium channel blockers: incidence and withdrawal rate-a meta-analysis of randomized trials. J Hypertens. 2011;29:1270-80.

37. Elliott WJ. Management of hypertension in the very elderly patient. Hypertension. 2004;44:800-4.

38. Wu ZB, Zhang Y, Yu QG, et al. Effects of valsartan combined with amlodipine or hydrochlorothiazide regimen on blood pressure variation in elderly hypertensive patients. Zhonghua Xin Xue Guan Bing Za Zhi. 2012;40:8-13. 